\title{
Sternal stress fracture in a gymnast: A case report and literature review
}

\author{
Ismail Hassan (MMed (Int Med), FCP (SA)) \\ Dimakatso Althea Ramagole (MB ChB, MSc (Sports Med)) \\ Dina Christina Janse van Rensburg (MB ChB, MMed (PhysMed), MSc (Sports Med)) \\ Catharina Cornela Grant (National Diploma in Analytical Chemistry, BSc, BSc (Hons), MSc) \\ Rob Collins MSc (Sports Med)
}

University of Pretoria, Division Sports Medicine (Centre for Sports Sciences)

\begin{abstract}
Introduction
There is a high incidence of injuries among gymnasts. ${ }^{1}$ This is not surprising given the highly repetitive nature of impacts associated with landings from dismounts and during floor routines. The majority of upper extremity stress fractures involve the distal radius. ${ }^{2}$ Sternal stress fractures are rare, with the only other similar type of injury being described in a 9-year-old gymnast who was training on the parallel bars when he sustained an acute posterior sterno-manubrial dislocation. ${ }^{3}$ Here is a description of a sternal stress fracture in a competitive gymnast.
\end{abstract}

\section{Case report}

A 15-year-old male competitive gymnast presented 1 week after routine training. While performing a floor exercise routine and following a tumble, he experienced central chest pain. As a result he withheld further training and consulted 1 week later, due to ongoing pain. There were no signs of ecchymosis or swelling over the sternum, while tenderness over the body of the sternum was present. The history of pain at rest suggested a fracture rather than bony bruising or a stress reaction. The absence of morning stiffness, which is an important symptom of costochondritis, made the diagnosis of costochondritis unlikely. In addition, the absence of ecchymosis and swelling excluded conditions like the Tietze's syndrome. Muscle weakness and skeletal deformities were not found on examination. The rest of the systemic examination was unremarkable.

Plain chest X-rays (Fig. 1) did not show any abnormalities and fractures could not be seen. The patient was referred for a bone scan which was indicative of a sternal stress fracture (Fig. 2a, 2b). The scan revealed increased uptake in the body of the sternum, exactly where the pain was located, demonstrating unequal uptake intensity strongly indicative of a stress fracture in the sternum.

The patient was treated symptomatically with oral analgesics and anti-inflammatory medication. Eight weeks after the onset of

\section{CORRESPONDENCE:}

Ismail Hassan

E-mail: ismail.hassan@ul.ac.za

Tel: 0764706620 symptoms the patient reported no pain and started to train again. Four months later he was pain free and fully active. He was also referred for advice on performance technique and education. He has, however, since been lost to follow-up.

\section{Discussion}

Stress fractures occur owing to repetitive muscular action on a bone. This repetitive stress causes periosteal resorption. Sternal stress fractures are rare, and account for only $0.5 \%$ of all sternal fractures. ${ }^{4}$ Only four cases have been reported in non-contact sport (Table I).

Upper extremity stress fractures in a gymnast involve the distal radius and scaphoid because of repetitive abduction and dorsiflex movement of the wrist. Forty five per cent

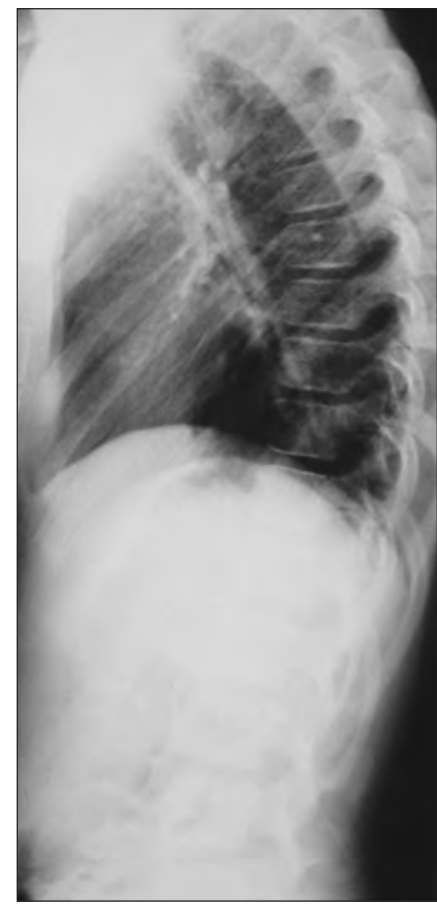

Fig. 1. Plain X-ray. of all stress fractures in a gymnast involve the pars interarticularis, because of considerable stress on the lower back as a result of repetitive flexion, hyperextension, rotation and compressive loading of the spine on landing. ${ }^{7,8}$

The mechanism of this stress fracture may be due to sudden forward angulations at the thoracic spine and violent protraction of the shoulders, stressing the sternum via the clavicles. We postulate that the forward flexion on the spine during the repeated tumbles and the motion on assuming the standing position contributed to the injury. The dynamic movement of sudden standing stresses the ribs and the maldistribution of forces across the scalene muscles and pectoralis major, impact on the sternum, resulting in the sternal stress fracture.

A common example of poor posture in gymnasts is excessive arching of the back. The manner in which the body is held during both static and dynamic movements can unduly stress sternal structures through maldistribution of forces and body weight over structures that are not suited to the various tasks. ${ }^{2,7}$ Sternal stress fractures heal with no sequelae and complications are rare. ${ }^{9}$ 


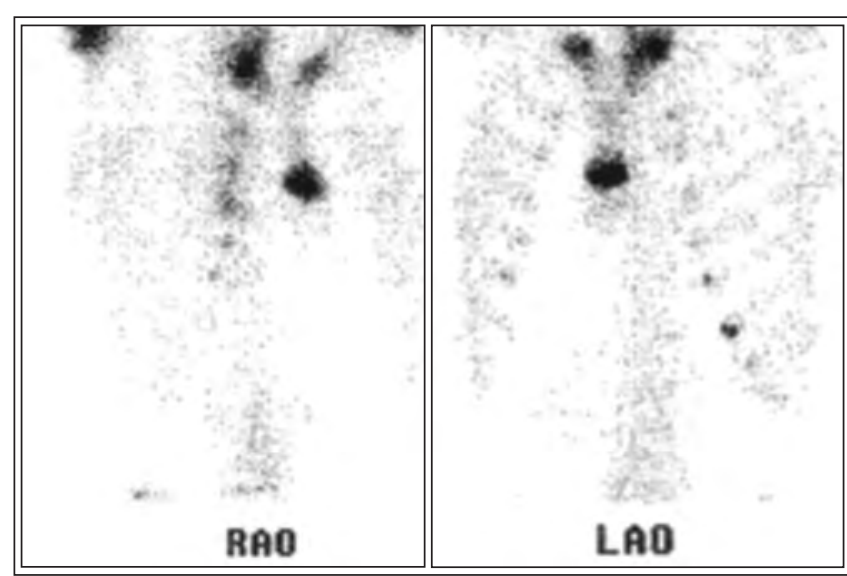

Figs $2 a$ and $b$. Right anterior oblique and left anterior oblique scans demonstrating unequal uptake intensity.

\begin{tabular}{|c|c|c|}
\hline Sternal stress fracture & Author & Sport \\
\hline 1 & Barbaix $1996^{5}$ & Golf \\
\hline 1 & Robertson $1996^{4}$ & Weight lifting \\
\hline 1 & McCurdie $1997^{6}$ & Triceps dips \\
\hline 1 & Robertson $1996^{4}$ & $\begin{array}{l}\text { Abdomen } \\
\text { muscle } \\
\text { training }\end{array}$ \\
\hline
\end{tabular}

Bone scans are sensitive in diagnosing stress fractures of the sternum and are reliable in detecting other bone pathologies, such as lymphoma (appearing as a cold signal) and multiple myeloma which usually has multiple lesions. Insufficiency fractures of the sternum have been reported in osteoporosis. This may be of concern in the adult population and adult women suffering from the 'female athlete triad'., 6,7

On the other hand, plain X-rays may not always be conclusive, while lateral X-ray views are superior to axial CT scanning and secondary signs of fracture such as retrosternal haematoma are specific but non-sensitive on CT. The CT scan does not demonstrate the horizontal fracture because of the axial direction. However, it readily demonstrates a retrosternal haematoma, without evidence of spread into the mediastinum - a specific sign of sternal injury (Fig. 3). A mediastinal haematoma could also contain venous or arterial blood, hence the importance of clinical cues. Due to the slice thickness and rotational cuts employed, the fracture goes unrecognised. ${ }^{10,11}$ MRI is the most sensitive modality overcoming this limitation, and also delineates better anatomical structures for other surrounding pathologies; however, it incurs a higher cost.

Ultrasound can also be used for diagnosing blunt chest trauma but is operator-dependent. It is useful when contemplating to infuse local anaesthetic into the site for pain relief. ${ }^{10}$ Unless their use is contraindicated, non-steroidal anti-inflammatory drugs (NSAIDs) are recommended for alleviation of pain and inflammation in impingement injuries, tenosynovitis and inflammatory arthropathy. ${ }^{12}$ However, NSAIDs have no effect on fracture healing, and their use in this setting is controversial and limited. Paracetamol is recommended for acute and chronic musculoskeletal pain. ${ }^{12}$ Absence from further training

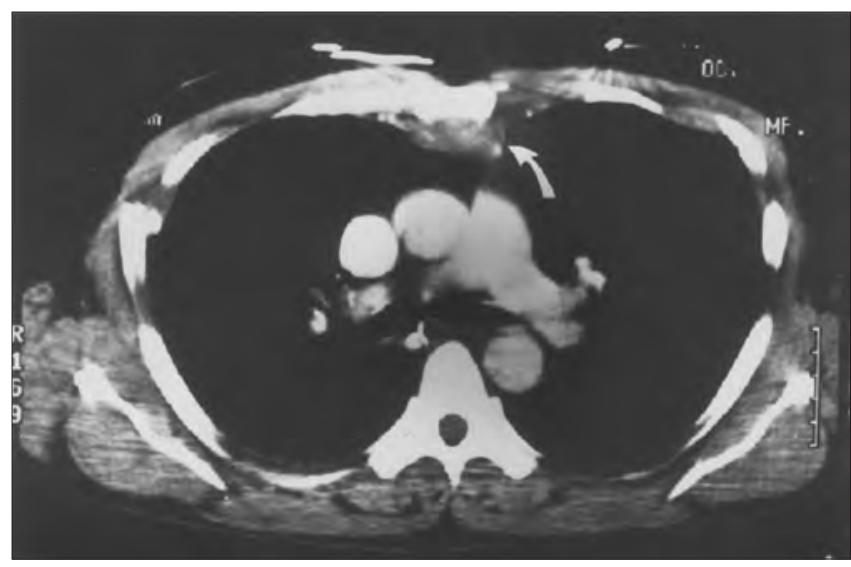

Fig. 3. An example of an axial CT scan.

is mandatory for healing and preventing complications. Preventive measures may incorporate neuromuscular training programmes to enhance proper skill mechanics. Equipment with safer design patterns for landing floor mats is recommended. This will facilitate better absorption of landing forces. ${ }^{2}$

\section{Conclusion}

- This case is in keeping with a sternal stress fracture. The majority of stress fractures in a gymnast occur during practice rather than competition and involve the lower extremities.

- Having excluded acute trauma and if bone injury is suspected, stress fractures should be entertained in the correct clinical context, even if signs and symptoms appear at unusual sites.

- Bone scintigraphy has a good application in the diagnosis of stress fracture in this setting.

\section{REFERENCES}

1. Caine DJ, Nassar L. Gymnastics injuries. Med Sport Sci 2005;48:1858.

2. Kolt G. Gymnastic injuries - why they occur. Austr Gymnast 1992;2:1819.

3. Nijs S, Broos O. Sterno-manubrial dislocation in a 9-year-old gymnast. Acta Chir Belg 2005;105:422-424.

4. Robertson K, Kristensen $\mathrm{O}$, Vejen L. Manubrium sterni stress fracture: an unusual complication of non-contact sport. $\mathrm{Br} J$ Sports Med 1996;30:176-177.

5. Barbaix EJ. Stress fractures of the sternum in a golf player. Int J Sports Med 1996;17:303-304

6. McCurdie I, Etherington J, Buchanan N. Sternal fracture in a female army officer cadet. Br J Sports Med 1997;31(2):164.

7. Dixon $M$, Fricker $P$. Injuries to elite gymnasts over $10 \mathrm{yrs}$. Med Sci Sports Exerc 1993;25(12):1322-1329.

8. Keller MS. Gymnastics injuries and imaging in children. Pediatr Radio 2009;39:1299-1306.

9. Chiu WC, D'amelio LE, Hammond JS. Sternal fractures in blunt chest trauma: a practical algorithm for management. Am J Emerg Med 1997;15:252-255.

10. Hugget JM, Rozler MH. CT findings of a sternal fracture. Injury 1998;29(8):623-626.

11. Anderson MW. Imaging of upper extremity stress fractures in the athlete. Clin Sports Med 2006;25:489-504.

12. Paoloni JA, Milne C, Orchard J, et al. Non-steroidal anti-inflammatory drugs in sports medicine: guidelines for practical but sensible use. $\mathrm{Br}$ Sports Med 2009;43:863-865. 